\title{
GAUGE EQUIVALENCE AND INVERSE SCATTERING FOR LONG-RANGE MAGNETIC POTENTIALS
}

\author{
GREGORY ESKIN AND HIROSHI ISOZAKI
}

\begin{abstract}
For Schrödinger operators with long-range magnetic vector potentials and short range electric scalar potentials in an exterior domain $\Omega$ in $\mathbf{R}^{n}$ with $n \geq 2$, we show that there is a one-to-one correspondence between the gauge equivalent classes of Hamiltonians and those of S-matrices, if $\Omega$ is exterior to a bounded convex obstacle.
\end{abstract}

\section{INTRODUCTION}

Let $\Omega=\mathbf{R}^{n} \backslash \overline{\mathcal{O}}$, where $\mathcal{O}$ is a bounded domain with smooth boundary, called an obstacle. We consider a Schrödinger operator

$$
H=\left(-\nabla_{x}-A(x)\right)^{2}+V(x)
$$

in $\Omega$ with Dirichlet boundary condition, where $A(x)=\left(A_{1}, \ldots, A_{n}\right) \in C^{\infty}(\bar{\Omega})$ is the magnetic vector potential and $V(x) \in C^{\infty}(\bar{\Omega})$ is the electric scalar potential. We assume that $A(x), V(x)$ are real-valued, and satisfy the following conditions: $V(x)$ is a short-range potential, i.e.

$$
\left|\partial_{x}^{\alpha} V(x)\right| \leq C_{\alpha}\langle x\rangle^{-1-\varepsilon_{0}-|\alpha|}, \quad \forall \alpha,
$$

where $\varepsilon_{0}>0$ and $\langle x\rangle=\left(1+|x|^{2}\right)^{1 / 2}, A(x)$ is a long-range potential, i.e.

$$
\left|\partial_{x}^{\alpha} A(x)\right| \leq C_{\alpha}\langle x\rangle^{-1-|\alpha|}, \quad \forall \alpha .
$$

Throughout the paper, $C$ 's denote various constants independent of the variable $x$ (and other parameters). We also assume that $A(x)$ satisfies the transversal gauge condition

$$
\left|\partial_{x}^{\alpha}(x \cdot A(x))\right| \leq C_{\alpha}\langle x\rangle^{-1-|\alpha|}, \quad \forall \alpha .
$$

It is well-known that the scattering operator $S$ exists under the conditions (1.2), (1.3), (1.4) (cf. [LoTh87]). The properties of $S$ related to the Aharonov-Bohm effect and the scattering problem were discussed in [AB59], [Rui83], [RoYa02], and others.

In this paper, we shall consider a slightly more restrictive class of long-range magnetic potentials than (1.3). Namely, in addition to the conditions (1.2), (1.3) and (1.4), we assume for some $R>0$

$$
A(x)=A_{0}(x)+A_{1}(x) \text { for }|x|>R,
$$

where $A_{0}(x) \in C^{\infty}\left(\mathbf{R}^{n} \backslash\{0\}\right)$, homogeneous of degree -1 , and satisfies

$$
x \cdot A_{0}(x)=0 .
$$

Date: December, 24, 2010. 
We also assume that $A_{1}(x)$ is a short-range potential decaying like (1.2). The condition (1.6) implies that $A_{1}(x)$ satisfies (1.4).

In order to define a gauge transformation group, we need to introduce two spaces of functions. Let $\mathcal{L}_{\infty, 0}$ be the set of real-valued functions $L(x) \in C^{\infty}(\bar{\Omega})$ satisfying

$$
\left|\partial_{x}^{\alpha} L(x)\right| \leq C_{\alpha}\langle x\rangle^{-\epsilon_{0}-|\alpha|}, \quad \forall \alpha,
$$

Let $\mathcal{H}_{0}$ be the set of $C^{\infty}\left(\mathbf{R}^{n} \backslash\{0\}\right)$-functions, homogeneous of degree 0 . We sometimes write $\psi(x) \in \mathcal{H}_{0}$ as $\psi(\hat{x}) \in C^{\infty}\left(S^{n-1}\right)$, where $\hat{x}=x /|x|$. When $n=2$, we also write $\varphi \in \mathcal{H}_{0}$ as $\varphi(\theta)$, where $\theta$ is the polar angle, i.e. $\hat{x}=(\cos \theta, \sin \theta)$.

Note that when $\varphi(x) \in \mathcal{H}_{0}$ the vector potential $\nabla_{x} \varphi(x)$ satisfies the transversal gauge condition

$$
x \cdot \nabla_{x} \varphi(x)=0 .
$$

Indeed, let $r=|x|$. Then $x \cdot \nabla_{x} \varphi(x)=r \frac{\partial}{\partial r} \varphi(\hat{x})=0$ since $\varphi(\hat{x})$ is independent of $r$.

Definition 1.1. Denote by $\mathbf{G}(\bar{\Omega})$ the group of $C^{\infty}(\bar{\Omega})$ functions having the following properties: $|g(x)|=1$ in $\bar{\Omega}$ and there exists a constant $R>1$ such that for $|x|>R$

$$
g(x)=\left\{\begin{array}{l}
e^{i(\psi(\hat{x})+L(x))}, \quad \psi \in \mathcal{H}_{0}, \quad L \in \mathcal{L}_{\infty, 0}, \quad \text { when } n \geq 3, \\
e^{i(m \theta+\varphi(\theta)+L(x))}, \quad \varphi \in \mathcal{H}_{0}, \quad L \in \mathcal{L}_{\infty, 0}, \quad \text { when } n=2,
\end{array}\right.
$$

where $m$ is an integer (which may depend on $g(x)$ ), and

$$
\int_{0}^{2 \pi} \varphi(\theta) d \theta=0
$$

We shall call $\mathbf{G}(\bar{\Omega})$ the gauge group and the transformation $u(x) \rightarrow g(x) u(x)$ the gauge transformation.

If $u(x)$ is the solution to the Schrödinger equation $H u=\lambda u, v(x)=g(x) u(x)$ satisfies $H^{\prime} v=\lambda v$, where

$$
H^{\prime}=\left(-i \frac{\partial}{\partial x}-A^{\prime}\right)^{2}+V^{\prime}
$$

with $V^{\prime}(x)=V(x)$, and

$$
A^{\prime}(x)=A(x)-i g^{-1} \frac{\partial g}{\partial x} .
$$

Magnetic potentials $A^{\prime}(x)$ and $A(x)$ satisfying (1.11) are called gauge equivalent. Let $\widehat{S}(\lambda)$ and $\widehat{S}^{\prime}(\lambda)$ be the scattering matrices corresponding to $H$ and $H^{\prime}$, respectively. Then (see, for example, [Ya06])

$$
\widehat{S}^{\prime}(\lambda)=e^{i \psi\left(D_{x}\right)} \widehat{S}(\lambda) e^{-i \psi\left(-D_{x}\right)}
$$

when $n \geq 3$, and

$$
\widehat{S}^{\prime}(\lambda)=e^{i m \theta\left(D_{x}\right)+i \varphi\left(D_{x}\right)} \widehat{S}(\lambda) e^{-i m \theta\left(-D_{x}\right)-i \varphi\left(-D_{x}\right)}
$$

when $n=2$. Scattering matrices $\widehat{S}(\lambda)$ and $\widehat{S}^{\prime}(\lambda)$ satisfying (1.12) or (1.13) are said to be gauge equivalent. Therefore, if the magnetic vector potentials are gauge equivalent, so are the scattering matrices. A natural question is the validity of the converse assertion. This is an inverse problem. Since the domain $\Omega$ could be 
multiply-connected, our task shoud be not only the recovery of the magnetic field but the recovery of the gauge equivalence classes of the electro-magnetic potentials.

In our previous work [EIO, we have dealt with the case $n=2$ (and $\mathcal{H}_{0} \ni$ $\varphi(\theta)=0$ ), and proven that if the scattering matrices coincide, the Schrödinger operators are gauge equivalent under the equal flux condition. Moreover, outside the convex obstacles, the equal flux condition is satisfied. Therefore, outside the convex obstacle, there is a one-to-one correspondence between the gauge equivalence classes of Schrödinger operators and those of S-matrices. The aim of this article is to extend this result to the case $n \geq 2$ including $\mathcal{H}_{0}$.

At the first sight, this problem seems to be of the topological nature, and the case with dimension $n \geq 3$ looks simpler than the case $n=2$. However, as it will be shown, results for $n=2$ and $n=3$ are more or less the same and they are determined by the principal part $A_{0}(x)$ of the long-range magnetic potential.

\section{INVERSE SCATTERING PROBLEM}

The following theorem is one of our main results.

Theorem 2.1. Let $n \geq 2$ and $H^{(j)}=\left(-i \frac{\partial}{\partial x}+H^{(j)}(x)\right)^{2}+V^{(j)}$ be two Schrödinger operators in $\Omega^{(j)}, j=1,2$, with Dirichlet boundary condition on $\partial \Omega^{(j)}$. Here $A^{(j)}=$ $A_{0}^{(j)}+A_{1}^{(j)}, j=1,2$, satisfy (1.5), (1.6), and $V^{(j)}(x)$ satisfy (1.2). Moreover, when $n=2$ we assume that

$$
\begin{aligned}
& \left|V^{(1)}(x)-V^{(2)}(x)\right| \leq C_{N}\langle x\rangle^{-N}, \quad \forall N, \\
& \left|A_{1}^{(1)}(x)-A_{1}^{(2)}(x)\right| \leq C_{N}\langle x\rangle^{-N}, \quad \forall N .
\end{aligned}
$$

Suppose $\widehat{S}^{(1)}(\lambda)=\widehat{S}^{(2)}(\lambda)$ for all $\lambda>0$ and suppose there exists $R>0$ such that $A_{0}^{(1)}(x)=A_{0}^{(2)}(x)$ for $|x|>R$. Then $\Omega^{(1)}=\Omega^{(2)}, V^{(1)}=V^{(2)}$ in $\Omega$ and $A^{(1)}$ and $A^{(2)}$ are gauge equivalent in $\Omega$.

Proof: Let $B_{R}=\{x:|x|<R\}$ be a ball that contains the complements to $\Omega_{1}$ and $\Omega_{2}$. Let $\left\{x_{0}+s \omega, s \in \mathbf{R}, \omega \in S^{n-1}\right\}$ be a line not intersecting $B_{R}$. It was shown in [Nic00], [We02], [BaWe07] that $\hat{S}^{(1)}(\lambda)=\hat{S}^{(2)}(\lambda)$ for all $\lambda>0$ implies

$$
\begin{gathered}
\exp \left(i \int_{-\infty}^{\infty} A^{(1)}\left(x_{0}+s \omega\right) \cdot \omega d s\right)=\exp \left(i \int_{-\infty}^{\infty} A^{(2)}\left(x_{0}+s \omega\right) \cdot \omega d s\right), \\
\int_{-\infty}^{\infty} V^{(1)}\left(x_{0}+s \omega\right) d s=\int_{-\infty}^{\infty} V^{(2)}\left(x_{0}+s \omega\right) d s
\end{gathered}
$$

Note that (1.6) implies that integrals in (2.3) converge. Since we assume that $A_{0}^{(1)}=A_{0}^{(2)}$ we get from (2.3) that

$$
\exp \left(i \int_{-\infty}^{\infty}\left(A_{1}^{(1)}-A_{1}^{(2)}\right)\left(x_{0}+s \omega\right) \cdot \omega d t\right)=1 .
$$

Therefore $\int_{-\infty}^{\infty}\left(A_{1}^{(1)}-A_{1}^{(2)}\right)\left(x_{0}+s \omega\right) \cdot \omega d t=2 \pi m$, where $m$ is integer. Since this integral tends to zero when $\left|x_{0}\right| \rightarrow \infty$ we get that $m=0$.

Consider first the case $n \geq 3$. Let $\Pi$ be an arbitrary two-dimensional plane in the exterior of $B$. Then it follows from (2.4), (2.5) (cf. [Nic00], [Es03], [BaWe07]) 
that $V^{(1)}=V^{(2)}$ on $\Pi$ and $\left.B_{1}^{(1)}\right|_{\Pi}=\left.B_{1}^{(2)}\right|_{\Pi}$, where $B_{1}^{(i)}=d A_{1}^{(i)}$ and $\left.B_{1}^{(1)}\right|_{\Pi}$ is the restriction of the two-form $B_{1}^{(i)}$ to $\Pi$. Since $\Pi$ is an arbitrary plane we get that

$$
V^{(1)}=V^{(2)}, \quad B_{1}^{(1)}=B_{1}^{(2)} \text { when }|x| \geq R .
$$

Therefore there exists $L_{1} \in \mathcal{L}_{\infty, 0}$ such that

$$
A_{1}^{(2)}-A_{1}^{(1)}=\nabla_{x} L_{1} \text { for }|x| \geq R,
$$

since $\mathbf{R}^{n} \backslash B_{R}$ is a simply-connected domain when $n \geq 3$.

In the case $n=2$ we get (2.6), (2.7) under the assumption (2.1), (2.2) (cf. [He99], [Nic00], [EIO]).

Extend arbitrarily $L_{1}(x)$ from $|x| \geq R$ to $\mathbf{R}^{n}, n \geq 2$. Make the gauge transformation of $H^{(2)}$ with the gauge $g_{1}(x)=e^{i L_{1}(x)}$. Then $H^{(1)}$ and $H^{(3)}=g_{1}^{-1} H^{(2)} g_{1}$ coincide for $|x| \geq R$. Note that $\widehat{S}^{(3)}(\lambda)=\widehat{S}^{(2)}(\lambda)$ since $\nabla_{x} L_{1}$ is a short-range potential. Now as in the proof of Theorem 5.7 in [EIO] we want to show that $\widehat{S}^{(1)}(\lambda)=\widehat{S}^{(2)}(\lambda)$ and $H^{(3)}=H^{(1)}$ for $|x| \geq R$ implies that the Dirichlet-toNeumann operators $\Lambda^{(1)}(\lambda)$ and $\Lambda^{(3)}(\lambda)$ for $H^{(1)}$ and $H^{(3)}$ respectively coincide on $C=\{|x|=R\}$ for any $\lambda>0$. Theorem 5.7 of [EIO] is not trivial in the longrange case. Fortunately, the results of $\S 2, \S 3,4.1,5.1$ of [EIO] hold for any $n \geq 2$ when conditions (1.2), (1.3), (1.4) are satisfied. In particular, we have the spatial asymptotics of the resolvent and also that of the eigenoperator, whose kernel is the distorted plane wave (cf. Lemma 3.9 and Theorems 4.4 and 4.5 in [EIO]). Next using the BC-method (cf. [Be97], [KuLa00], [Es06], [Es07]) we get that $\Lambda^{(1)}(\lambda)=\Lambda^{(2)}(\lambda)$ for any $\lambda>0$ implies that $\Omega^{(1)}=\Omega^{(2)}, V^{(1)}=V^{(2)}$ in $\Omega$ and $A^{(2)}-A^{(1)}=i g^{-1} \nabla_{x} g$ in $\Omega$ (cf. Theorem 5.8 in [EIO]).

Theorem 2.1proves the main result under the additional assumption $A_{0}^{(1)}=A_{0}^{(2)}$. In the next two sections we show that this condition is necessary when $H^{(1)}$ and $H^{(2)}$ are gauge equivalent and it follows from the equality of the scattering matrices $\widehat{S}^{(1)}(\lambda)=\widehat{S}^{(2)}(\lambda)$ in the case when $\Omega^{(1)}=\Omega^{(2)}$, and it is an exterior to a convex obstacle.

\section{The TWO-Dimensional CASE}

We first consider the 2-dimensional case. Let us start with the following fact.

Lemma 3.1. Let $A_{0}(x) \in C^{\infty}\left(\mathbf{R}^{2} \backslash\{0\}\right)$, and $A_{0}(x)$ is homogeneous of degree -1 . Suppose $A_{0}(x) \cdot x=0$. Then there exists $a_{0}(x) \in \mathcal{H}_{0}$ such that

$$
\begin{gathered}
A_{0}(x)=\alpha \frac{\left(-x_{2}, x_{1}\right)}{|x|^{2}}+\nabla_{x} a_{0}(x), \\
\alpha=\frac{1}{2 \pi} \int_{|x|=R} A_{0}(x) \cdot d x, \quad \int_{0}^{2 \pi} a_{0}(\cos \theta, \sin \theta) d \theta=0 .
\end{gathered}
$$

Proof: Let $A_{0}(x)=\left(A_{01}(x), A_{02}(x)\right)$. Then $A_{0}(x) \cdot x=0$ implies $x_{1} A_{01}+$ $x_{2} A_{02}=0$, and

$$
\frac{A_{02}}{x_{1}}=\frac{A_{01}}{-x_{2}}=\frac{a(x)}{|x|^{2}}
$$


where $a(x)$ is homogeneous of degree zero. Therefore

$$
A_{01}=-\frac{x_{2}}{|x|^{2}} a(x), \quad A_{02}=\frac{x_{1}}{|x|^{2}} a(x) .
$$

Since $a(x)=a(x /|x|)$ we have in polar coordinates $x_{1}=r \cos \theta, x_{2}=r \sin \theta$ that $a(x /|x|)=a(\cos \theta, \sin \theta)$. Let $\hat{a}(\theta)=a(\cos \theta, \sin \theta)$, i.e. $\hat{a}(\theta)$ is $2 \pi$-periodic function of $\theta$, and let

$$
\alpha=\frac{1}{2 \pi} \int_{0}^{2 \pi} \hat{a}(\theta) d \theta
$$

Note that $\alpha$ in (3.2), (3.4) is the same. Moreover, $\alpha$ can be defined as the limit: $\alpha=\lim _{R \rightarrow \infty} \frac{1}{2 \pi} \int_{|x|=R} A(x) \cdot d x$.

Let $b(\theta)=\hat{a}(\theta)-\alpha$. Then $\int_{0}^{2 \pi} b(\theta) d \theta=0$. Therefore $b(\theta)$ can be represented in the form

$$
b(\theta)=\frac{\partial}{\partial \theta} \hat{a}_{0}(\theta)
$$

where $\hat{a}_{0}(\theta)$ is $2 \pi$-periodic and

$$
\int_{0}^{2 \pi} \hat{a}_{0}(\theta) d \theta=0
$$

Let $a_{0}(x /|x|) \in \mathcal{H}_{0}$ be such that $a_{0}(\cos \theta, \sin \theta)=\hat{a}_{0}(\theta), \theta=\tan ^{-1}\left(x_{1} / x_{2}\right)$. We have

$$
\begin{gathered}
\frac{\partial a_{0}}{\partial x_{1}}=\frac{\partial \hat{a}_{0}(\theta)}{\partial \theta} \frac{\partial \theta}{\partial x_{1}}=\frac{x_{2}}{|x|^{2}} \frac{\partial \hat{a}_{0}(\theta)}{\partial \theta}, \\
\frac{\partial a_{0}}{\partial x_{2}}=\frac{\partial \hat{a}_{0}(\theta)}{\partial \theta} \frac{\partial \theta}{\partial x_{2}}=-\frac{x_{1}}{|x|^{2}} \frac{\partial \hat{a}_{0}(\theta)}{\partial \theta} .
\end{gathered}
$$

Therefore we have proven

$$
\begin{aligned}
A_{0}(x) & =\frac{\left(-x_{2}, x_{1}\right)}{|x|^{2}} \hat{a}(\theta)=\frac{\left(-x_{2}, x_{1}\right)}{|x|^{2}}\left(\alpha+\frac{\partial \hat{a}_{0}(\theta)}{\partial \theta}\right) \\
& =\alpha \frac{\left(-x_{2}, x_{1}\right)}{|x|^{2}}+\nabla_{x} a_{0}(x) .
\end{aligned}
$$

It follows from Lemma 3.1 that when $n=2$ any magnetic potential of the form (1.5), (1.6) is gauge equivalent in $\Omega$ to the Aharonov-Bohm type potential

$$
A_{\alpha}=\alpha \frac{\left(-x_{2}, x_{1}\right)}{|x|^{2}}+A_{1}^{\prime}(x)
$$

where $A_{1}^{\prime}$ is a short-range potential.

It was shown in [Ya06], [RoYa02] that the kernel of scattering matrix $\widehat{S}_{\alpha}(\lambda)$ corresponding to (3.6) has the form

$$
S_{\alpha}\left(\theta, \theta^{\prime}\right)=S_{\alpha}\left(\theta-\theta^{\prime}\right)+S_{\alpha}^{\prime}\left(\theta, \theta^{\prime}\right)
$$

where

$$
\begin{gathered}
S_{\alpha}(\theta)=\cos (\alpha \pi) \delta(\theta)+i \frac{\sin (\alpha \pi)}{\pi} \text { p.v. } \frac{e^{i[\alpha] \theta}}{1-e^{i \theta}}, \\
\left|S_{\alpha}^{\prime}\left(\theta, \theta^{\prime}\right)\right| \leq C\left|\theta-\theta^{\prime}\right|^{-\delta}, 0<\delta<1,
\end{gathered}
$$


$[\alpha]$ is the integer part of $\alpha$. (In [Ya06], [RoYa02] the case $\Omega=\mathbf{R}^{2}$ was considered. The extension to the case of domains with obstacles was shown in [EIO]).

It follows from Lemma 3.1 and (1.13) that the kernel of the scattering matrix $\widehat{S}(\lambda)$ corresponding to $A(x)=A_{0}+A_{1}$ has the form (cf. [RoYa02], [Ya06])

$$
S\left(\theta, \theta^{\prime}\right)=e^{i \hat{a}_{0}(\theta)-i \hat{a}_{0}\left(\pi+\theta^{\prime}\right)}\left(S_{\alpha}\left(\theta-\theta^{\prime}\right)+S_{\alpha}^{\prime}\left(\theta, \theta^{\prime}\right)\right) .
$$

The next theorem shows that in the case of a convex obstacle $\hat{S}^{(1)}(\lambda)=\hat{S}^{(2)}(\lambda)$ implies that $A_{0}^{(1)}=A_{0}^{(2)}$ if $\alpha$ is not an integer.

Theorem 3.2. Let $H^{(j)}=\left(-i \frac{\partial}{\partial x}+A^{(j)}\right)^{2}+V^{(j)}$ be two Schrödinger operators in the same domain $\Omega \subset \mathbf{R}^{2}$, where $\Omega$ is the exterior to a convex obstacle. Suppose $V^{(j)}, j=1,2$, are short-range potentials and

$$
A^{(j)}=\alpha_{j} \frac{\left(-x_{2}, x_{1}\right)}{|x|^{2}}+\nabla_{x} \varphi_{j}(x)+A_{1}^{(j)}, \quad j=1,2,
$$

where $\varphi_{j}(x) \in \mathcal{H}_{0}, \int_{S^{1}} \varphi_{j}(\hat{x}) d \sigma=0, \quad A_{1}^{(j)}$ are short-range potentials, $j=1,2$. Suppose $\widehat{S}^{(1)}(\lambda)=\widehat{S}^{(2)}(\lambda)$ for all $\lambda>0$ and $\alpha_{1}$ is not integer. Suppose also that (2.1), (2.2) hold. Then

$$
\alpha_{1}=\alpha_{2}, \quad \varphi_{1}(x)=\varphi_{2}(x),
$$

$V^{(1)}=V^{(2)}$ in $\Omega$ and there exists $L_{1}(x) \in \mathcal{L}_{\infty, 0}$ such that $A_{1}^{(2)}-A_{1}^{(1)}=\nabla_{x} L_{1}$.

Proof: As has been proved in [Nic00], [We02], $\widehat{S}^{(1)}(\lambda)=\widehat{S}^{(2)}(\lambda)$ for all $\lambda>$ 0 implies (2.3), (2.4) for all lines $x=x_{0}+s \omega$ not intersecting $\Omega$. Let $\omega=$ $(\cos \theta, \sin \theta), x_{1}=x_{10}+s \cos \theta, x_{2}=x_{20}+s \sin \theta, x_{0} \cdot \omega=0$. Then

$$
\begin{array}{r}
\int_{-\infty}^{\infty} \alpha \frac{\left(-x_{2}, x_{1}\right)}{|x|^{2}} \cdot \omega d s=\alpha \int_{-\infty}^{\infty} \frac{\left|x_{0}\right|}{\left|x_{0}\right|^{2}+s^{2}} d s=\alpha \pi, \\
\int_{-\infty}^{\infty} \frac{\partial \varphi\left(x_{0}+s \omega\right)}{\partial x} \cdot \omega d s=\int_{-\infty}^{\infty} \frac{d}{d s} \varphi\left(x_{0}+s \omega\right) d s \\
=\left.\varphi\left(x_{0}+s \omega\right)\right|_{-\infty} ^{\infty}=\left.\varphi\left(\frac{1}{|s|} x_{0}+\frac{s}{|s|} \omega\right)\right|_{-\infty} ^{\infty}=\varphi(\omega)-\varphi(-\omega),
\end{array}
$$

where we used that $\varphi$ is homogeneous of degree 0 . Note that $\int_{-\infty}^{\infty} A_{1}^{(j)}\left(x_{0}+s \omega\right)$. $\omega d s \rightarrow 0$ when $\left|x_{0}\right| \rightarrow \infty, x_{0} \cdot \omega=0$. Therefore taking the limit in (2.3) when $\left|x_{0}\right| \rightarrow \infty$ we get

$$
\left(\alpha_{2}-\alpha_{1}\right) \pi+\varphi(\omega)-\varphi(-\omega)=2 \pi m,
$$

for some integer $m$, where $\varphi(\omega)=\varphi_{2}(\omega)-\varphi_{1}(\omega)$. It follows from (3.14) that $\varphi(\omega)-\varphi(-\omega)$ does not depend on $\omega$. Since $\varphi(\omega)-\varphi(-\omega)$ is odd, there exists $\omega_{0}$ such that $\varphi\left(\omega_{0}\right)-\varphi\left(-\omega_{0}\right)=0$. Therefore

$$
\begin{gathered}
\varphi(\omega)-\varphi(-\omega)=0, \quad \forall \omega \in S^{1}, \\
\alpha_{2}-\alpha_{1}=2 m .
\end{gathered}
$$

It follows from (3.14) and (2.3) that (2.5) holds. Therefore using (2.4), (2.5), (2.1) and (2.2), we get as in the proof of Theorem 2.1 (cf. [Nic00], [BaWe07], [Es03]) that $V^{(1)}=V^{(2)}$ in $\Omega$ and (2.7) holds in $\Omega$. As in the proof of Theorem 
2.1. let $H^{(3)}=g_{1}^{-1} H^{(2)} g_{1}$ where $g_{1}=e^{i L_{1}}$. Let $\widehat{S}^{(3)}(\lambda)$ is the scattering matrix corresponding to $H^{(3)}$. Note also that $H^{(3)}$ has the form

$$
H^{(3)}=\left(-i \frac{\partial}{\partial x}+\alpha_{2} \frac{\left(-x_{2}, x_{1}\right)}{|x|^{2}}+\nabla_{x} \varphi_{2}+A_{1}^{(1)}\right)^{2}+V^{(1)}(x) .
$$

Let $g(x)=e^{i 2 m \theta+i \varphi(\hat{x})}$ where $\hat{x}=(\cos \theta, \sin \theta)$. Then $g^{-1} H^{(3)} g=H^{(1)}$. Therefore (cf. (1.13) )

$$
S^{(3)}\left(\theta, \theta^{\prime}\right)=e^{i 2 m\left(\theta-\theta^{\prime}\right)+i \varphi(\omega)-i \varphi(-\omega)} S^{(1)}\left(\theta, \theta^{\prime}\right),
$$

where (cf. (3.7), (3.8))

$$
\begin{gathered}
S^{(1)}\left(\theta, \theta^{\prime}\right)=\left(S_{\alpha_{1}}\left(\theta-\theta^{\prime}\right)+S_{\alpha_{1}}^{\prime}\left(\theta, \theta^{\prime}\right)\right) e^{i \varphi_{1}(\omega)-i \varphi_{1}(-\omega)}, \\
\left|S_{\alpha_{1}}^{\prime}\left(\theta, \theta^{\prime}\right)\right| \leq C\left|\theta-\theta^{\prime}\right|^{-\delta}, \quad 0 \leq \delta<1 .
\end{gathered}
$$

Since $\widehat{S}^{(1)}=\widehat{S}^{(3)}$ we get

$$
\left(e^{i 2 m\left(\theta-\theta^{\prime}\right)+i(\varphi(\omega)-\varphi(-\omega))}-1\right) S^{(1)}\left(\theta, \theta^{\prime}\right)=0 .
$$

Denote $\varphi_{0}(\theta)=\varphi(\cos \theta, \sin \theta)$. Then $\varphi(-\cos \theta,-\sin \theta)=\varphi(\cos (\theta+\pi), \sin (\theta+\pi))=$ $\varphi_{0}(\theta+\pi)$. We have $\varphi_{0}(\theta)-\varphi_{0}(\theta+\pi)=0, \forall \theta$. Using the Taylor formula we get

$$
\begin{aligned}
\varphi_{0}(\theta)-\varphi_{0}\left(\theta^{\prime}+\pi\right) & =\varphi_{0}\left(\theta^{\prime}\right)+\varphi_{0}^{\prime}\left(\theta^{\prime}\right)\left(\theta-\theta^{\prime}\right)+O\left(\left(\theta-\theta^{\prime}\right)^{2}\right)-\varphi_{0}\left(\theta^{\prime}+\pi\right) \\
& =\varphi_{0}^{\prime}\left(\theta^{\prime}\right)\left(\theta-\theta^{\prime}\right)+O\left(\left(\theta-\theta^{\prime}\right)^{2}\right) .
\end{aligned}
$$

Since $\alpha_{1}$ is not an integer we have for arbitrary $\theta_{0}^{\prime}$ :

$$
\left|S^{(1)}\left(\theta, \theta^{\prime}\right)\right|=\left|S_{\alpha_{1}}\left(\theta-\theta^{\prime}\right)+S_{\alpha_{1}}^{\prime}\left(\theta, \theta^{\prime}\right)\right| \geq C\left|\theta-\theta_{0}^{\prime}\right|^{-1}
$$

for all $0<\theta-\theta^{\prime}<\varepsilon, \varepsilon$ is small. Take $\theta_{0}^{\prime}$ such that $\varphi_{0}^{\prime}\left(\theta_{0}^{\prime}\right)=0$. Then using (3.21) and assuming $m \neq 0$ we get

$$
\left|e^{i 2 m\left(\theta-\theta_{0}^{\prime}\right)+i\left(\varphi_{0}\left(\theta_{0}^{\prime}\right)-\varphi_{0}\left(\theta_{0}^{\prime}+\pi\right)\right)}-1\right| \geq C\left|\theta-\theta_{0}^{\prime}\right| .
$$

Therefore the left-hand side of (3.20) is nonzero for $0<\theta-\theta_{0}^{\prime}<\varepsilon$. This contradiction proves that $2 m=\alpha_{2}-\alpha_{1}=0$.

Assuming that $\varphi_{0}(\theta)$ is not zero we can find $\theta_{0}^{\prime \prime}$ such that $\varphi^{\prime}\left(\theta_{0}^{\prime \prime}\right) \neq 0$. Then taking into account that $m=0$ we get

$$
\left|e^{i\left(\varphi_{0}(\theta)-\varphi_{0}\left(\theta_{0}^{\prime \prime}+\pi\right)\right)}-1\right| \geq C\left|\theta-\theta_{0}^{\prime \prime}\right| \text { for }\left|\theta-\theta_{0}^{\prime \prime}\right| \text { small. }
$$

Again (3.24) and (3.22) contradict (3.20). Therefore $\varphi_{0}^{\prime}(\theta)=0$ for all $\theta$ and hence $\varphi_{0}(\theta)=\varphi_{2}(\theta)-\varphi_{1}(\theta)=0, \forall \theta$.

The following theorem shows that the condition $A_{0}^{(1)}=A_{0}^{(2)}$ is necessary if one assumes that $\widehat{S}^{(1)}(\lambda)=\widehat{S}^{(2)}(\lambda)$ and $H^{(1)}$ and $H^{(2)}$ are gauge equivalent.

Theorem 3.3. Let $\Omega$ be an arbitrary domain with obstacles. Let $H^{(j)}=\left(-i \frac{\partial}{\partial x}+\right.$ $\left.A^{(j)}(x)\right)^{2}+V^{(j)}(x)$ be the Schrödinger operators in $\Omega$ as in Theorem [2.1. Suppose $\widehat{S}^{(1)}(\lambda)=\widehat{S}^{(2)}(\lambda)$ and suppose that $H^{(1)}$ and $H^{(2)}$ are gauge equivalent, i.e. there exists $g(x) \in \mathbf{G}(\bar{\Omega})$ such that $H^{(1)}=g^{-1} H^{(2)} g$. Suppose also that

$$
\alpha_{1}=\lim _{R \rightarrow \infty} \frac{1}{2 \pi} \int_{|x|=R} A^{(1)}(x) \cdot d x
$$

is not an integer. Then $A_{0}^{(1)}(x)=A_{0}^{(2)}(x)$. 
Proof: Denote $H^{(3)}=g^{-1} H^{(2)} g$. Then $H^{(3)}=H^{(1)}$ and therefore $\widehat{S}^{(3)}(\lambda)=$ $\widehat{S}^{(1)}(\lambda)$. It follows from (1.13) that

$$
S^{(3)}\left(\theta, \theta^{\prime}\right)=e^{i m\left(\theta-\theta^{\prime}\right)+i\left(\varphi_{0}(\theta)-\varphi_{0}(\theta+\pi)\right)} S^{(2)}\left(\theta, \theta^{\prime}\right) .
$$

Since $S^{(2)}\left(\theta, \theta^{\prime}\right)=S^{(1)}\left(\theta, \theta^{\prime}\right)$ we get the same equation as in (3.20):

$$
\left(e^{i 2 m\left(\theta-\theta^{\prime}\right)+i\left(\varphi_{0}(\theta)-\varphi_{0}(-\theta+\pi)\right)}-1\right) S^{(1)}\left(\theta, \theta^{\prime}\right)=0,
$$

i.e. we are in the same situation as in the proof of Theorem 3.2. Therefore $m=$ $0, \varphi_{0}(\theta)=0$. Hence $A_{0}^{(2)}=A_{0}^{(1)}$.

On the basis of Theorem 3.2 we can formulate our ultimate result in 2-dimensions.

Theorem 3.4. Let $\Omega$ be the exterior of a convex obstacle. Let $H^{(j)}, j=1,2$, be the same as in Theorem 3.2. Let $\widehat{S}^{(j)}(\lambda)$ be the scattering matrices corresponding to $H^{(j)}, j=1,2$. If $\widehat{S}^{(1)}(\lambda)$ and $\widehat{S}^{(2)}(\lambda)$ are gauge equivalent for all $\lambda>0$, then $V^{(1)}=V^{(2)}$ and $A^{(1)}$ and $A^{(2)}$ are gauge equivalent.

Proof: Since $\widehat{S}^{(1)}(\lambda)$ and $\widehat{S}^{(2)}(\lambda)$ are gauge equivalent there exists $g(x)=$ $e^{i m \theta+i \varphi_{0}(\theta)}$ such that (1.13) holds. Let $H^{(3)}=g^{-1} H^{(2)} g$. Then $\widehat{S}^{(1)}(\lambda)=\widehat{S}^{(3)}(\lambda)$ where $\widehat{S}^{(3)}(\lambda)$ is the scattering matrix for $H^{(3)}$. Also we have that $A_{0}^{(3)}$ is gauge equivalent to $A_{0}^{(2)}, A_{1}^{(3)}=A_{1}^{(2)}, V^{(3)}=V^{(2)}$. Since $\widehat{S}^{(3)}(\lambda)=\widehat{S}^{(1)}(\lambda)$ we get applying Theorem 3.2 that $A_{0}^{(3)}=A_{0}^{(1)}, V^{(3)}=V^{(1)}$ and $A_{1}^{(3)}-A_{1}^{(1)}=\nabla_{x} L_{1}$. Therefore $A^{(2)}=A_{0}^{(2)}+A_{1}^{(2)}$ is gauge equivalent to $A^{(1)}=A_{0}^{(1)}+A_{1}^{(1)}$ and $V^{(1)}=V^{(2)}$.

We end this section with a conjecture that Theorem 3.4 holds for arbitrary domain with obstacles.

\section{THE CASE $n \geq 3$}

The following theorem is the analog of Theorem 3.2 for $n \geq 3$.

Theorem 4.1. Let $\Omega$ be the exterior of a convex obstacles in $\mathbf{R}^{n}, n \geq 3$. Let $H^{(j)}=\left(-i \frac{\partial}{\partial x}-A^{(j)}\right)^{2}+V^{(j)}(x)$ be two Schrödinger operators as in Theorem 2.1. Suppose curl $A_{0}^{(1)} \neq 0$, and that $\widehat{S}^{(1)}(\lambda)=\widehat{S}^{(2)}(\lambda)$ for all $\lambda>0$. Then $V^{(1)}=V^{(2)}$, $A_{1}^{(2)}-A_{1}^{(1)}=\nabla_{x} L_{1}$ with $L_{1} \in \mathcal{L}_{\infty, 0}$ and $A_{0}^{(1)}=A_{0}^{(2)}$.

Proof: Consider (2.3), (2.4). Let $A_{0}=A_{0}^{(2)}-A_{0}^{(1)}, A_{1}=A_{1}^{(2)}-A_{1}^{(1)}$. It follows from $(2.3) \int_{-\infty}^{\infty}\left(A_{0}+A_{1}\right)\left(x_{0}+s \omega\right) \cdot \omega d s=2 \pi m$ where $m \in \mathbf{Z}$. As in Theorem 2.1 take any two-dimensional plane $\Pi$ in $\Omega$. The proof in [Es03], pp 990-991, give that $\left.B\right|_{\Pi}=0$ even when $m \neq 0$. Here $B=B_{0}+B_{1}, B_{0}=d A_{0}, B_{1}=d A_{1}, B$ is the restriction of 2 -form $B$ to $\Pi$. Since $\Pi$ is arbitrary we have as in Theorem 2.1 that $B_{0}+B_{1}=0, V^{(1)}=V^{(2)}$ in $\Omega$. Note that $B_{0}(x)=b(x) /|x|^{2}$ where $b(x)$ is homogeneous of degree zero and $\left|B_{1}(x)\right| \leq C\langle x\rangle^{-2-\varepsilon_{0}}$. Multiplying $B_{0}+B_{1}=0$ by $|x|^{2}$ and taking the limit when $|x| \rightarrow \infty$ we get that $b(x)=0$. Therefore $B_{0}=0$ and $B_{1}=0$. Since $\Omega$ is a simply connected domain, we have $A_{1}=\nabla_{x} L_{1}, A_{0}=\nabla_{x} \varphi$, where $L_{1} \in \mathcal{L}_{\infty, 0}$ and $\varphi(x) \in \mathcal{H}_{0}$. Since $\int_{-\infty}^{\infty} \nabla_{x} L_{1} \cdot \omega d s=0$, we get from (2.3) that $\int_{-\infty}^{\infty} A_{0} \cdot \omega d s=2 \pi m$. 
Therefore as in (3.13)

$$
2 \pi m=\int_{-\infty}^{\infty} \frac{\partial \varphi}{\partial x}\left(x_{0}+s \omega\right) \cdot \omega d s=\int_{-\infty}^{\infty} \frac{d}{d s} \varphi\left(x_{0}+s \omega\right) d s=\varphi(\omega)-\varphi(-\omega) .
$$

Changing $\omega$ to $-\omega$ we get $m=0$, i.e. $\varphi(\omega)-\varphi(-\omega)=0, \forall \omega \in S^{n-1}$. Let $g(x)=$ $e^{i \varphi(\omega)+i L_{1}(x)}$ and let $H^{(3)}=g^{-1} H^{(2)} g$. Then $H^{(3)}=H^{(1)}$ and therefore $\widehat{S}^{(3)}=\widehat{S}^{(1)}$. By (1.12) we have $\widehat{S}^{(3)}\left(\omega, \omega^{\prime}\right)=\widehat{S}^{(2)}\left(\omega, \omega^{\prime}\right) e^{i \varphi(\omega)-i \varphi\left(-\omega^{\prime}\right)}$. Since $\widehat{S}^{(1)}=\widehat{S}^{(2)}$ we have

$$
\left(e^{i\left(\varphi(\omega)-\varphi\left(-\omega^{\prime}\right)\right)}-1\right) S^{(1)}\left(\omega, \omega^{\prime}\right)=0 .
$$

As in the proof of Theorem 3.2, we have by the Taylor formula

$$
e^{i\left(\varphi(\omega)-\varphi\left(-\omega^{\prime}\right)\right)}=e^{i\left(\varphi_{x}\left(\omega^{\prime}\right) \cdot\left(\omega-\omega^{\prime}\right)+O\left(\left|\omega-\omega^{\prime}\right|^{2}\right)\right.},
$$

since $\varphi\left(\omega^{\prime}\right)-\varphi\left(-\omega^{\prime}\right)=0$. Therefore if $\omega_{0}^{\prime}$ is such that $\varphi_{x}\left(\omega_{0}^{\prime}\right) \neq 0$ then

$$
\left|e^{i\left(\varphi(\omega)-\varphi\left(-\omega^{\prime}\right)\right.}-1\right| \geq C\left|\omega-\omega^{\prime}\right|,
$$

when $\left|\omega-\omega^{\prime}\right|$ is small, $\omega^{\prime}$ is close to $\omega_{0}^{\prime}$ and $\left(\omega-\omega^{\prime}\right) \cdot \varphi_{x}\left(\omega_{0}^{\prime}\right) \neq 0$. Since curl $A_{0}^{(1)} \neq 0$, we get that $\nabla_{x} I(x, \omega) \neq 0$, where $I(x, \omega)=\int_{-\infty}^{\infty} A_{0}^{(1)}(x+s \omega) \cdot \omega d s$. Then it follows from [Ya06] that $S^{(1)}\left(\omega, \omega^{\prime}\right)$ contains a principal value integral and therefore $S^{(1)}\left(\omega, \omega^{\prime}\right) \neq 0$ when (4.4) holds. This contradicts (4.2). Therefore we must have $\varphi=0$, i.e. $A_{0}^{(1)}=A_{0}^{(2)}$.

The proof of the following Theorems are the same as that of Theorems 3.3 and 3.4 with the reference to Theorem 3.2 replaced by the reference to Theorem 4.1

Theorem 4.2. Suppose $\Omega_{1}=\Omega_{2}=\Omega$ is an arbitrary domain with obstacles in $\mathbf{R}^{n}, n \geq 3$, and $H^{(1)}$ and $H^{(2)}$ are the same as in Theorem 4.1. Suppose curl $A_{0}^{(1)} \not \equiv$ 0. If $\widehat{S}^{(1)}(\lambda)=\widehat{S}^{(2)}(\lambda)$ and if $H^{(1)}$ and $H^{(2)}$ are gauge equivalent, then $A_{0}^{(1)}=A_{0}^{(2)}$.

Theorem 4.3. Suppose $\Omega_{1}=\Omega_{2}=\Omega \subset \mathbf{R}^{n}, n \geq 3$, where $\Omega$ is the exterior of a convex obstacle. Let $H^{(j)}=\left(-\frac{\partial}{\partial x}+A^{(j)}\right)^{2}+V^{(j)}, \widehat{S}^{(j)}(\lambda), j=1,2$, be the same as in Theorem 4.1. If $\widehat{S}^{(1)}(\lambda)$ and $\widehat{S}^{(2)}(\lambda)$ are gauge equivalent for all $\lambda>0$, then $H^{(1)}$ and $H^{(2)}$ are gauge equivalent.

It follows from Theorem 4.2 that the assertion: $\widehat{S}^{(1)}(\lambda)=\widehat{S}^{(2)}(\lambda)$ implies $A_{0}^{(1)}=$ $A_{0}^{(2)}$ is necessary when $H^{(1)}$ and $H^{(2)}$ are gauge equivalent. This leads us to a conjecture that Theorem 4.1 and consequently Theorem 4.3 are true for any obstacle.

\section{REFERENCES}

[AB59] Y. Aharonov and D. Bohm, Significance of electromagnetic potential in the quantum theory, Phys. Rev. 115 (1959), 485-491.

[BaWe07] M. Ballesteros and R. Weder, High-velocity estimates for the scattering operator and the Aharonov-Bohm effect in three dimensions, Arxiv 0711.256.

[Be97] M. I. Belishev, Boundary control in reconstruction of manifolds and metrics (the BC method), Inverse Problems 13 (1997), R1-R45.

[BeKu92] I.M. Belishev and Y. Kurylev, The reconstruction of the Riemannian manifold via its spectral data, Comm. in P. D. E. 17 (1992), 767-804.

[ES03] G. Eskin, Inverse problems for the Schrödinger operators with electromagnetic potentials in domains with obstacles, Inverse Problems 19 (2003), 985-996.

[Es06] G. Eskin, A new approach to hyperbolic inverse problems, Inverse Problems 22 (2006), 815-831. 
[Es07] G. Eskin, A new approach to hyperbolic inverse problems II (Global step), Inverse Problems 23 (2007), 2343-2356.

[EIO] G. Eskin, H. Isozaki and S. O'dell, Gauge equivalence and inverse scattering for AharonovBohm effect, Commun. in P. D. E. 35 (2010), 2164-2194.

[He99] S. Helgason, The Radon transform, 2nd edition, Birkhäuser, Boston-Basel-Berlin (1999).

[H085] L. Hörmander, Analysis of Linear Partial Differential Operators IV, Springer, Berlin (1985)

[Is01] H. Isozaki, Asymptotic properties of solutions to 3-particle Schrödinger equations, Comm. Math. Phys 222 (2001), 371-413

[IsKi85] H. Isozaki and H. Kitada, Modified wave operators with time-dependent modifiers, J. Fac. Sci. Univ. Tokyo 32 (1985), 77-104.

[KaKuLa01] A. Katchalov, Y. Kurylev and M. Lassas, Inverse Boundary Spectral Problems, Chapman and Hall/CRC 123 (2001).

[KuLa00] Y. Kurylev and M. Lassas, Hyperbolic inverse problems with data on a part of the boundary, AMS/IP, Stud. Adv. Math. 16 (2000), 259-272.

[LoTh87] M. Loss and B. Thaler, Scattering of particles by long-range magnetic fields, Ann. Phys. 176 (1987), 159-180

[Nic00] F. Nicoleau, Scattering matrices for Schrödinger operators in the presence of magnetic field. The Aharonov-Bohm phenomenon, J. Math. Phys. 41 (2000), 5223-5237.

[PeTo89] M. Peshkin and A. Tonomura, The Aharanov-Bohm Effect, Lect. Notes in Phys. 340, Springer-Verlag, Berlin (1989).

[RoYa02] Ph Roux and D. Yafaev, On the mathematical theory of the Aharonov-Bohm effect, J. Phys. A: Math. Gen. 35 (2002), 7481-7492.

[RoYa03] Ph Roux and D. Yafaev, The scattering matrix for the Schrödinger operator with a long-range electro-magnetic potential, J. Math. Phys. 44 (2003), 2762-2786.

[Rui83] S.N.M. Ruijsenaars, The Aharonov-Bohm effect and scattering theory, Annals pf Phys. 146 (1983), 1-34.

[TOTTEYY] A. Tonomura, N. Osakabe, T. Matsuda, T. Kawasaki, J. Endo, S. Yano, and H. Yamada, Evidence for Aharonov-Bohm effect with magnetic field completely shielded from electron wave, Phys. Rev. Lett. 56 (1986), 792.

[We02] R. Weder, The Aharonov-Bohm effect and time-dependent inverse scattering theory, Inverse Problems 18 (2002), 1041-1056.

[Ya03] D. Yafaev, High energy and smoothness asymptotic expansion of scattering amplitude, J. Funct. Anal. 202 (2003), 526-570

[Ya06] D. Yafaev, Scattering by magnetic fields, St.Petersburg Math. J. 17 (20060, 675-695 J. Funct. Anal. 202 (2003), 526-570

Department of mathematics, UCLA, Los Angeles, CA 90095-1555, USA

Institute of Mathematics, University of Tsukuba, Tsukuba, 305-8571, Japan 